\title{
Experience of severe desaturation during anesthetic induction period in an obese adult patient with Prader-Willi syndrome -A case report-
}

\author{
Joon Woo Choi, Eun-Ju Kim, Byung Woo Min, Jong Seouk Ban, Sang Gon Lee, and Ji-Hyang Lee \\ Department of Anesthesiology and Pain Medicine, Daegu Fatima Hospital, Daegu, Korea
}

Prader-Willi syndrome is characterized by infantile hypotonia, childhood-onset obesity, short stature, mental retardation, hyperphagia, hypogonadism. After infantile hypotonia phase, patient is prone to morbid obesity due to hyperphagia. Complications associated with morbid obesity are recognized as the main risk factors for death the lifespan of patients with Prader-Willi syndrome. We experienced desaturation and bronchospasm during arteriovenous fistula surgery in an obese adult with Prader-Willi syndrome. (Korean J Anesthesiol 2012; 62: 179-183)

Key Words: Anesthetic management, Desaturation, Morbid obesity, Prader-Willi syndrome.

Prader-Willi Syndrome (PWS) is a genetic disorder which characteristically features hypotonia, obesity, short stature, mental retardation, hyperphagia, hypogonadism, hypogenitalism and short life span [1]. Clinically, it can be divided into infancy characterized by hypotonia and childhood characterized by hyperphagia and obesity [2]. There are not many reports regarding the anesthetic management of adult PWS patients with a shortened life span as a complication of morbid obesity [3-5], and there are practically no reports regarding airway management during anesthetic induction. A 26 year-old PWS patient with morbid obesity, sleep apnea, hypertension, diabetic mellitus and chronic renal failure presented severe desaturation and bronchospasm during emergence from anesthesia despite sufficient preoxygenation and upper body elevation of 40 degrees during anesthesia induction. Thus, we report about the anesthetic management of adult PWS patients with morbid obesity, accompanied by a literature review.

\section{Case Report}

A 26 year-old male patient visited the hospital with rash, pain, and edema in the left foot. He was hospitalized and treated for a diagnosis of cellulitis in the left foot. At the time of hospitalization, his vital signs were as follows: body temperature $38.2^{\circ} \mathrm{C}$, heart rate 110 beats $/ \mathrm{min}$, blood pressure $140 / 70 \mathrm{mmHg}$ and respiration rate 22 times/min. In his medical history, he had hypotonia, poor sucking, and cryptorchidism at birth. While he was an infant, he was hospitalized several times for pneumonia, and diagnosed with PWS after confirmation

Received: June 3, 2011. Revised: July 5, 2011. Accepted: July 26, 2011.

Corresponding author: Eun-Ju Kim, M.D., Department of Anesthesiology and Pain Medicine, Daegu Fatima Hospital, 576-31, Shinam-dong, Dong-gu, Daegu 701-600, Korea. Tel: 82-53-940-7434, Fax: 82-53-954-7417, E-mail: kej1127@fatima.or.kr

(C) This is an open-access article distributed under the terms of the Creative Commons Attribution Non-Commercial License (http:// creativecommons.org/licenses/by-nc/3.0/), which permits unrestricted non-commercial use, distribution, and reproduction in any medium, provided the original work is properly cited. 
of long arm deletion of the 15th chromosome. When he was 12 years old, he was diagnosed with level 2 mental retardation and diabetes. He took medication, but the diabetes was not controlled, and obesity progressed due to hyperphagia. He was diagnosed with chronic renal failure 3 years ago and was receiving hemodialysis three times a week.

The treatment progress for the cellulitis was not satisfactory so amputation of the foot was advised but only skin disinfection and antibiotic treatment were done due to the refusal of the guardian. While on conservative therapy, the patient developed an obstruction in their right brachiobasilic arteriovenous fistula. Ultrasonography (USG) was done, and he was diagnosed with several giant pseudoaneurysms. Aneurysmectomy and vessel ligation were done under local anesthesia. At the time, the patient showed anxiety so the guardian was in the operating room to stabilize the patient while the surgery was done. While maintaining hemodialysis by doing a dual lumen catheterization in the right subclavian vein, left brachiocephalic fistula surgery was planned. Due to problems with the patient being uncooperative and time, the operating surgeon requested general anesthesia.

In the blood test before surgery, hemoglobin $(\mathrm{Hb})$ was 7.5 $\mathrm{g} / \mathrm{dl}$; hematocrit was $22.8 \%$; the glomerular filtration rate was $11.65 \mathrm{ml} / \mathrm{min}$; sodium was $131 \mathrm{mEq} / \mathrm{l}$; potassium $\left(\mathrm{K}^{+}\right)$was $6.6 \mathrm{mEq} / \mathrm{l}$; and glucose was $112-354 \mathrm{mg} / \mathrm{dl}$. According to the ABGA, pH was confirmed as 7.311; $\mathrm{PaCO}_{2}$ was $36.0 \mathrm{mmHg} ; \mathrm{PaO}_{2}$ was $70.9 \mathrm{mmHg}$; $\mathrm{HCO}_{3}{ }^{-}$was $17.8 \mathrm{mEq} / \mathrm{l}$; and $\mathrm{SaO}_{2}$ was $93.0 \%$. There were no abnormalities in the EKG, and according to the chest X-ray, even though pulmonary edema was observed at the time of the hospital visit, it had improved, and cardiomegaly

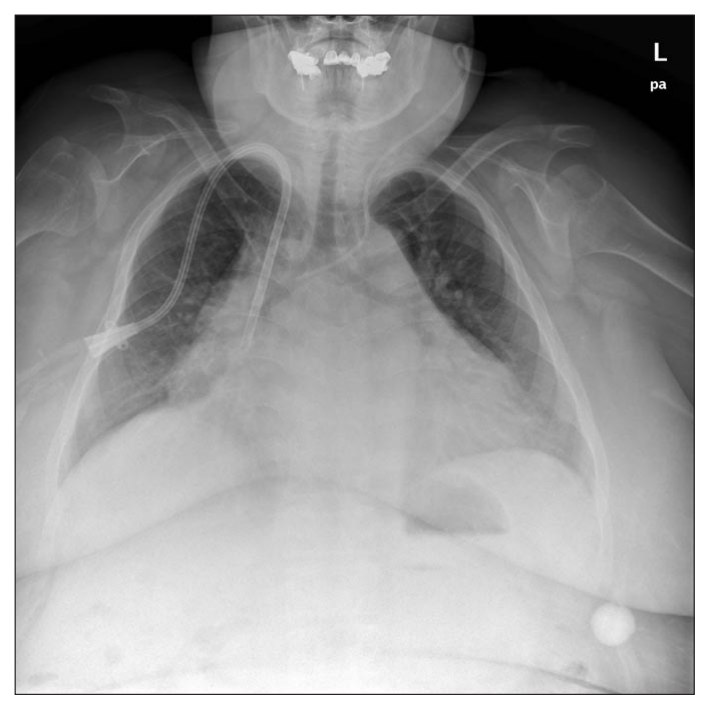

Fig. 1. Preoperative chest X-ray shows cardiomegaly, dual lumen catheterization and central vein catheterization in the morbidly obese patient. was observed (Fig. 1). In the visit before the surgery, the patient had a height of $150 \mathrm{~cm}$, a weight of $105 \mathrm{~kg}$, a body mass index (BMI) of $46.7 \mathrm{~kg} / \mathrm{m}^{2}$ with morbid obesity, and a short neck and limbs. Airway management was anticipated to be difficult with mild sleep apnea, but he was observed to be Mallampatti class II. Most of the time, he was in a sitting position and received oxygen treatment only when he felt it hard to breathe. Due to his mental retardation, only simple communication was possible. Before entering the operating room, $\mathrm{H}_{2}$ antagonist was administered as premedication.

Upon arrival at the operating room, his vital signs were as follows: body temperature $37.5^{\circ} \mathrm{C}$, heart rate 85 beats $/ \mathrm{min}$, blood pressure 160/90 $\mathrm{mmHg}$, and respiration rate 22 times/ min. A blood pressure cuff (M1576A, Agilent Technologies, Santa Clara, USA) with a diameter of $42-54 \mathrm{~cm}$ was bound on his right ankle for noninvasive blood pressure measurement, but severe edema in the lower limbs prevented measurement for more than 10 minutes so it was decided to carry out invasive arterial pressure measurement. USG (S-Nerve ${ }^{\mathrm{TM}}$, Sonosite, Bothell, USA) guided arterial catheterization was done on the right dorsalis pedis artery, but due to edema, the 20 gauge catheter could not reach the artery. In the same way, arterial catheterization was done on the right radial artery successfully. The blood pressure measured through the invasive arterial pressure was $190 / 90 \mathrm{mmHg}$. $\mathrm{SaO}_{2}$ measured after entering the operating room was $95 \%$, and during the invasive arterial pressure measurement, $100 \%$ oxygen was administered through a mask and semi-closed circuit system for 25 minutes before anesthesia with upper body elevation of 40 degrees. For endotracheal intubation, remifentanil $0.06 \mu \mathrm{g} / \mathrm{kg} / \mathrm{min}$ was administered and propofol $120 \mathrm{mg}$ was injected. After loss of consciousness $\mathrm{SaO}_{2}$ rapidly dropped to lower than $90 \%$ so mask ventilation using both hands was done. After checking that the tidal volume was $250 \mathrm{ml}$ and the end tidal carbon dioxide concentration $\left(\mathrm{EtCO}_{2}\right)$ was about $20-30 \mathrm{mmHg}$, atracurium $50 \mathrm{mg}$ was administered. We had thought that mask ventilation would be sufficient but the $\mathrm{SaO}_{2}$ recovery did not reach our expectations; thus, we decided to do endotracheal intubation. After confirming the disappearance of spontaneous respiration, intubation was attempted with a $7.5 \mathrm{~mm}$ diameter endotracheal tube. Here, the $\mathrm{SaO}_{2}$ was about $77 \%$. He was grade 1 on the Cormack and Lehane classification where the vocal cords were easily visible, but the vocal cords were small compared to his age so intubation failed since the tube could not enter. After failing at the endotracheal intubation the first time, the $\mathrm{SaO}_{2}$ decreased to $58 \%$ so mask ventilation was done. After increasing the $\mathrm{SaO}_{2}$ to $82 \%$, endotracheal intubation succeeded with a 7.0 $\mathrm{mm}$ diameter tube, but in the interim, the $\mathrm{SaO}_{2}$ decreased to $70 \%$. Using pressure control ventilation, the airway pressure was adjusted to $30 \mathrm{mmHg}$; the respiration rate was 15 per minute, 
and the fractional inspired $\mathrm{O}_{2}$ concentration $\left(\mathrm{FiO}_{2}\right)$ was at $50 \%$, but tidal volume was measured as $400 \mathrm{ml}$; $\mathrm{EtCO}_{2}$ was $42 \mathrm{mmHg}$; and $\mathrm{SaO}_{2}$ was $93 \%$, so the $\mathrm{FiO}_{2}$ was adjusted to $70 \%$ and positive end expiratory pressure was started with $5 \mathrm{cmH}_{2} \mathrm{O}$. To prevent laryngeal spasm, dexamethasone $10 \mathrm{mg}$ was IV injected, and sevoflurane $2.0 \mathrm{vol} \%$ and remifentanil $0.03 \mu \mathrm{g} / \mathrm{kg} / \mathrm{min}$ was used to maintain anesthesia. Afterwards, $\mathrm{SaO}_{2}$ was maintained at 93-98\%. In the first blood test after anesthesia, it was confirmed that $\mathrm{Hb}$ was $8.8 \mathrm{~g} / \mathrm{dl} ; \mathrm{K}^{+}$was $5.4 \mathrm{mEq} / \mathrm{l}$, and glucose was $77 \mathrm{mg} /$ dl so $50 \%$ dextrose in $100 \mathrm{ml}$ of water was combined with insulin 5 UNIT and IV injected at $10 \mathrm{ml} / \mathrm{h}$. One hour after the start of the surgery, spontaneous breathing returned so atracurium 20 mg was additionally administered. The total operative time was 3 hours and 45 minutes, and vital signs during anesthesia were maintained at stable state with a systolic blood pressure of 100$115 \mathrm{mmHg}$, diastolic blood pressure of $45-60 \mathrm{mmHg}$, and a heart rate of $75-85$ beats $/ \mathrm{min}$. Arterial blood was collected every hour to check ABGA, electrolytes, and glucose levels. In the final blood test, it was confirmed that $\mathrm{Hb}$ was $7.8 \mathrm{~g} / \mathrm{dl} ; \mathrm{K}^{+}$was $5.8 \mathrm{mEq} / \mathrm{l}$; glucose was $96 \mathrm{mg} / \mathrm{dl} ; \mathrm{PaCO}_{2}$ was $52.5 \mathrm{mmHg}$; and $\mathrm{PaO}_{2}$ was $110 \mathrm{mmHg}$, and during surgery, $350 \mathrm{ml}$ of $5 \%$ dextrose in water was administered. As the spontaneous breathing of the patient returned after surgery, the tidal volume was maintained at about $300 \mathrm{ml}$, and once in a while, the patient exhaled deeply and vital capacity was measured up to $800 \mathrm{ml}$. The patient's state of consciousness was not clear, but as spontaneous breathing became regular and the patient opened his eyes and showed discomfort and behavior of trying to pull out the intubated tube, extubation was done. After extubation, progress was observed with assistance from an oxygen mask. Although chest movements were good, the $\mathrm{SaO}_{2}$ decreased down to $88 \%$. When listening to both chests, expiratory wheezing could be heard so inhalation therapy with steroids and $\beta_{2}$ agonist was immediately started. After inhalation therapy, expiratory wheezing disappeared, and respirations were stable thus the patient was moved to the recovery room. In the recovery room, the patient was observed for 30 minutes with oxygen administered at $5 \mathrm{~L} /$ min through a mask with the upper body elevated at about $40^{\circ}$ and $\mathrm{SaO}_{2}$ was maintained at $95 \%$ so the patient was moved to the intensive care unit. The chest X-ray checked in the intensive care unit showed no difference from the one before the surgery. On the first day after surgery during sleep, the $\mathrm{SaO}_{2}$ decreased to lower than $90 \%$; therefore, oxygen treatment was done, and afterwards, vital signs were stable so 2 days later the patient was moved to the general ward.

\section{Discussion}

PWS is the most common cause for syndromic obesity, and the frequency of occurrence is about $1 / 25,000$ [2]. This syn- drome is known to be caused by paternal interstitial deletion of the long arm proximal part of the 15th chromosome, maternal uniparental disomy, abnormalities in the imprinting process and by mutations [6]. Clinically, it can be divided into infancy, which is characterized by severe hypotonia and childhood which is characterized by obesity from hyperphagia. In infancy, respiratory infection or asphyxia can occur from severe hypotonia, poor sucking, and weakened coughing and crying while in childhood from age 1-6 after recovery of hypotonia, hyperphagia develops leading to morbid obesity. A short stature, scoliosis, sleep disorder, thermoregulation disorder and emotional disorders may also occur [6].

For all ages, the annual death rate is $3 \%$, but for over 30 years of age, the death rate reaches $7 \%$ [2]. PWS patients generally die early from complications related to obesity, such as diabetic mellitus, hypertension, sleep apnea, cardiovascular disease, and respiratory failure [6]. Nishikawa et al. [7] reported respiratory failure that developed from morbid obesity and sleep apnea in a 19 year-old male PWS patient with a height of 150 $\mathrm{cm}$, a weight of $140 \mathrm{~kg}$, and a BMI of $62.2 \mathrm{~kg} / \mathrm{m}^{2}$. After this patient underwent mechanical ventilation and lost $50 \mathrm{~kg}$ from caloric restriction, his respiratory failure improved and successful extubation was possible. Morbid obesity in PWS patients can greatly influence pulmonary function so it is an area that needs to be carefully managed before, during, and after anesthesia. Cho et al. [3] reported desaturation in a PWS patient with accompanying sepsis during anesthetic induction. In this case, mask control ventilation was not suitable due to micrognathia, and it was believed that obesity and sepsis would have affected the situation. In our case, the patient was morbidly obese with a BMI of $40 \mathrm{~kg} / \mathrm{m}^{2}$, and in the pulmonary function test, restrictive lung disease was confirmed. When lying down, dyspnea developed from increased abdominal pressure so most of the time, the patient was in a sitting position. Before anesthesia, a sufficient amount of oxygen was administered using a mask but after loss of consciousness, there was a rapid decline in $\mathrm{SaO}_{2}$. Although mask ventilation using both hands was relatively working, the $\mathrm{SaO}_{2}$ did not recover to more than $90 \%$. In morbidly obese patients who are sedated or under anesthesia, the excessively increased abdominal pressure decreases lung volume; functional residual capacity decreases significantly; alveolar-arterial oxygenation gradient increases, and there is an increase in respiratory resistance [8]. In addition, due to morbid obesity, the metabolic rate is high so the oxygen demand also increases, which is thought to lead to rapid desaturation. The authors thought that this desaturation can be recovered through mask ventilation and administered muscle relaxant, but afterwards, it was thought to be a poor decision that could have caused critical desaturation in the patient if endotracheal intubation had been impossible. In the 
case of patients with morbid obesity, severe desaturation can be expected despite sufficient oxygen administration before anesthesia and proper mask ventilation thus rapid-sequence intubation (RSI) or awake intubation can help in preventing hypoxia. In preoperative airway assessment, laryngoscopy and bronchoscopy examination as well as physical examination should be done. If endotracheal intubation is expected to be relatively easy, RSI can be planned while awake intubation can be planned for cases where endotracheal intubation is expected to be difficult. However, as in our case, it can be anticipated that cooperation for awake intubation will be difficult from a PWS patient with mental retardation and is sensitive to NPO. In addition, for the mechanical ventilation of our patient, a high airway pressure was expected so in our first attempt, a $7.5 \mathrm{~mm}$ diameter tube used, but entry failed because the vocal cords were too small. There have been reports that relatively smaller diameter tubes can be used when the vocal cords are small compared to the patients' age [4], but in obese patients, using the largest possible tube can reduce airway pressure; therefore, there is some difficulty when selecting the tube size. In cases of endotracheal intubation of obese PWS patients, always prepare various sized tubes beforehand, and after checking the size of the vocal cords, it is important to quickly select the appropriate tube to succeed in the first attempt. Additionally, when endotracheal intubation is difficult, it is safe practice to have an intubating laryngeal mask airway prepared beforehand.

It is called metabolic syndrome if more than three risk factors such as obesity, hypertension, diabetic mellitus, and dyslipidemia are present, and this is a strong indicator for atherosclerotic cardiovascular disease. Brambilla et al. [9] compared an obese PWS patient group, non-obese PWS patient group and obese but non-PWS control group for the presence of metabolic syndrome. Metabolic syndrome was not found in the non-obese PWS patient group but found in both the obese PWS patient group and the obese but non-PWS control group. Metabolic syndrome was more related to the condition of obesity rather than that of PWS; therefore, complications were not found in non-obese PWS patients who were diagnosed early and received growth hormone therapy or dietary control. In the case of PWS patients with accompanying metabolic syndrome, a thorough evaluation of cardiac function should be done before anesthesia. In our case, there were no abnormalities in the EKG, but cardiomegaly was observed in the chest x-ray and the guardian refused ultrasonocardiography.

In PWS patients, sufficient consideration should be given when selecting medications. During infancy, there are domestic reports that succinylcholine has been used as a muscle relaxant due to its short effective time since there is severe hypotonia in infants with PWS [10]. After infancy, hypotonia is mostly recovered, but there have been reports that muscle relaxants that are effective for a long time should be avoided [11]. In the case of our patient, hyperkalemia from chronic renal failure was observed so succinylcholine was not used, and atracurium was administered instead. Contrary to expectations, an hour after surgery, spontaneous breathing was restored so additional atracurium was administered, and directly after the end of the surgery, spontaneous breathing was quickly restored so recovery from muscle relaxants was similar to the response of average adults. There were two candidates for the maintenance of anesthesia, desflurane and sevoflurane since they accumulate less in fat cells and patients recover from them quickly. Among those two, sevoflurane was selected because of the following effects: less irritation on the airways, its bronchodilative effects, and repression of hypoxic pulmonary vasoconstriction [12]. During anesthesia, there were no specific problems other than high airway pressure and extubation was done after complete awakening. However, bronchospasm developed and it was treated with inhalation therapy using steroids and $\beta_{2}$ agonist. In premedication, the saliva secretion of the PWS patient was reduced so glycopyrrolate was not used [6].

In patients with morbid obesity, there are difficulties to IV catheterization and non-invasive blood pressure measurements. Kumar and Chuan [13] reported that the arterial approach using USG heightens the success rate of the catheterization while reduces the complications due to a reduced number of attempts. In the case of our patient, it was difficult to IV catheterization so with cooperation from radiology, USG guided central vein catheterization was done on the left internal jugular vein. The only available place for noninvasive blood pressure measurement was the right lower limb but measurements were not possible due to obesity and edema. Arterial catheterization was attempted on the right dorsalis pedis artery but it was confirmed through USG images that the 20 gauge arterial catheter had not reached the artery. Thus, USG guided arterial catheterization was done on the right radial artery for invasive blood pressure measurement.

In PWS patients, morbid obesity increases the abdominal pressure, which increases the occurrence rate of hiatal hernia along with the risk of aspiration pneumonia [5]. In our patient, after premedication with $20 \mathrm{mg}$ of $\mathrm{H}_{2}$ antagonist, endotracheal intubation was done with a suction apparatus ready for use. During endotracheal intubation, gastric secretion or foreign substances were not observed. In addition, it is difficult for PWS patients to adhere to fasting times due to mental retardation, temper tantrums and hyperphagia. As the fasting time became longer before general anesthesia, our patient had adverse reactions to our medical staff such as screaming, but under the supervision of the guardian, the fasting time was observed. Previously, there had been a history of anxiety where cooperation was not possible without the guardian during 
surgery under local anesthesia.

Palmer and Atlee [14] reported that PWS patients use circulating glucose in the synthesis of fat even on an empty stomach so relatively high levels of glucose should be maintained for the main organs to function. In our case, the glucose level was maintained at $110-350 \mathrm{mg} / \mathrm{dl}$ with insulin treatment; at the start of the surgery, it was $77 \mathrm{mg} / \mathrm{dl}$ so dextrose in water was continually injected to raise the glucose level to $96 \mathrm{mg} / \mathrm{dl}$. No symptoms related to hypoglycemia were observed in the operating room or recovery room.

There are difficulties in controlling the body temperature of PWS patients. Hypothermia and hyperthermia can all occur, and although functional disorders in the hypothalamus are presumed to be the cause, more research is needed. This abnormality in body temperature control is not characteristic of PWS but commonly accompanies defects in neurodevelopment [15]. The body temperature of our patient was maintained at $37.5^{\circ} \mathrm{C}$, but it was difficult to discern abnormalities in body temperature control due to the cellulitis on the left foot. Other than this, scoliosis, epilepsy, and arrhythmia can accompany PWS; thus, caution should be taken in airway management and when selecting medication according to the accompanying disease.

In conclusion PWS can be clinically divided into two periods, and after childhood, it is necessary to assess for morbid obesity and accompanying complications. In cases of patients who failed to control for hyperphagia, thorough examination should be done for cardiovascular disease, respiratory failure, hypertension, and diabetic mellitus that can occur from morbid obesity, while also checking for accompanying disorders such as body temperature control disorder, scoliosis, epilepsy, arrhythmia and IV catheterization. In addition, in our case, we experienced respiratory failure and rapidly progressing desaturation in the patient due to morbid obesity despite sufficient preoxygenation and mask ventilation. Preoperative evaluation of airway should be thoroughly done to plan for either RSI or awake intubation, while tubes of various sizes and a laryngeal mask should be prepared for safe anesthetic induction.

\section{References}

1. Holm VA, Cassidy SB, Butler MG, Hanchett JM, Greenswag LR, Whitman BY, et al. Prader-Willi syndrome: consensus diagnostic criteria. Pediatrics 1993; 91: 398-402.

2. Whittington JE, Holland AJ, Webb T, Butler J, Clarke D, Boer H. Population prevalence and estimated birth incidence and mortality rate for people with Prader-Willi syndrome in one UK Health Region. J Med Genet 2001; 38: 792-8.

3. Cho EC, Jee SE, Jang Y, Park SS, Kim JT, Song HK. Prader-Willi syndrome; a case report. Korean J Anesthesiol 1999; 36: 1091-4.

4. Cavaliere F, Cormaci S, Cormaci M, Alberti A, Colabucci F. General anesthesia in Prader-Willi syndrome. Minerva Anestesiol 1996; 62: 327-32.

5. Sloan TB, Kaye CI. Rumination risk of aspiration of gastric contents in the Prader-Willi syndrome. Anesth Analg 1991; 73: 492-5.

6. Cassidy SB, Driscoll DJ. Prader-Willi syndrome. Eur J Hum Genet 2009; 17: 3-13.

7. Nishikawa M, Mizutani T, Nakao T, Kamoda T, Takahashi S, Toyooka H. Respiratory failure due to morbid obesity in a patient with Prader-Willi syndrome: an experience of long-term mechanical ventilation. J Anesth 2006; 20: 300-3.

8. Pelosi P, Croci M, Ravagnan I, Cerisara M, Vicardi P, Lissoni A, et al. Respiratory system mechanics in sedated, paralyzed, morbidly obese patients. J Appl Physiol 1997; 82: 811-8.

9. Brambilla $\mathrm{P}$, Crinò A, Bedogni G, Bosio L, Cappa M, Corrias A, et al. Metabolic syndrome in children with Prader-Willi syndrome: the effect of obesity. Nutr Metab Cardiovasc Dis 2011; 21: 269-76.

10. Kang K, Park SE, Cho YW, Kim CH, Park SH. Prader-Willi syndrome. Korean J Anesthesiol 2004; 46: 751-4.

11. Dearlove OR, Dobson A, Super M. Anaesthesia and Prader-Willi syndrome. Paediatr Anaesth 1998; 8: 267-71.

12. Doi M, Takahashi T, Ikeda K. Respiratory effects of sevoflurane used in combination with nitrous oxide and surgical stimulation. J Clin Anesth 1994; 6: 1-4.

13. Kumar A, Chuan A. Ultrasound guided vascular access: efficacy and safety. Best Pract Res Clin Anaesthesiol 2009; 23: 299-311.

14. Palmer SK, Atlee JL 3rd. Anesthetic management of the Prader-Willi syndrome. Anesthesiology 1976; 44: 161-3.

15. Williams MS, Rooney BL, Williams J, Josephson K, Pauli R. Investigation of thermoregulatory characteristics in patients with Prader-Willi syndrome. Am J Med Genet 1994; 49: 302-7. 